\title{
Acercamiento al papel de los procesos de exclusión social y su relación con la salud*
}

\section{Approach to the role of the social exclusion processes and their relation with health}

\author{
Dra. María Raquel Agost Felip, ${ }^{\mathrm{I}}$ DraC. Libertad Martín Alfonso ${ }^{\mathrm{II}}$ \\ I Universidad Jaume I. Castellón de La Plana, España. \\ II Escuela Nacional de Salud Pública. La Habana, Cuba.
}

\begin{abstract}
RESUMEN
El presente trabajo pr esenta un acercamiento desde la perspectiva teórica al análisis del modelo de Determinantes Sociales de la Salud de la OM S (2005) en el que se destacan los determinantes estructura les, y dentro de estos el papel de los procesos de exclusión social como aspectos a tener en cuenta por su repercusión en la salu d de gru pos vulnerables. Se pr etende argum entar la compresión del problema a partir de la presentación de conceptos y modelos de exclusión social y exclusión en salud, lo qu e contribuirá a la v ez al in cremento del con ocimiento del tema para los interesados.
\end{abstract}

Palabras clav e: Determinantes sociales, exclusió n social, exclusión en salud, exclusión social en salud.

\section{ABSTRACT}

The present paper presented an approa ch to the analysis of the So cial Determinants in Health model of the Worl d Health Organization from a theoreti cal perspective. It underlin ed the str uctural determinants and the rol e of the soci al exclusion processes as factors to be ta ken into con sideration becau se of their impact on the most vulnerable g roups' health. It was intended to su pport the understanding of this problem through the submission of concept $\mathrm{s}$ and model $\mathrm{s}$ of 
social exclusi on and heal th e xclusion, which wi II contri bute to expa nd the knowledge of those people interested in this topic.

Key words: Social determinants, social exclusion, health exclusion, social exclusion in health.

\section{INTRODUCCIÓN}

La humanidad ha experimentado rápidas y profundas transformaciones en todas las esferas de la v ida eco nómica, cu Itural, social y polí tica. La gl obalización y sus consecuencias macroeconómicas influyen en el aumento de las desigualdades entre los países y los gr upos sociales, incr ementa los conflictos, disminuye las posibilidades de empleo, la $s$ oport unidades y el $r$ espeto a los de rechos huma nos. Estas condiciones repercuten en el campo de la salud y aumenta la responsabilidad de la sociedad para garantizar los cuidados a toda la población.

La crisis económica a nivel mundial provoca un debilitamiento de los esfuerzos para enfrentar p or parte del Estado, de modo colectivo, los problemas de salud y ha agudizado la llamada "crisis de la salu d pú blica". P ara su el iminación se $\mathrm{h}$ an propuesto, desde hace varios años, di ferentes aportes apunta ndo haci a la necesidad de nuevos paradigmas en la comprensión y solución de los problemas de salud en el terreno teórico y práctico. ${ }^{1}$

La reflexión en torno a los problem as de la salu d pú blica con temporánea propone que se an alice de ma nera crít ica y prof unda los det erminantes de la cr isis y se realicen proposiciones de políticas gl obales de salud que a ctualicen sus concepciones y prácticas. Diversas inicia tivas deben estar enfocadas a la formación avanzada e n salud pública, al perfeccionamiento de lo $\mathrm{s}$ recursos humanos y a la proyección de investigaciones e intervenciones dir igidas a mejorar e instaurar paulatinamente una nueva experiencia en este campo. ${ }^{1}$

En dicho marco se inserta actualmente el enfoque de análisis de los Determinantes Sociales de la Sa lud (DSS). En est e se in tenta in tegrar los dif erentes $n$ iveles y dimensiones desde los cuales se debe actuar para resolver muchos problemas que están en la base de las manifestaciones de las inequidades en salud. La perspectiva de trabajo con los DSS está respaldada po $r$ la Declaración de la Comisión de DSS de la Organización Mundial de la Salud (OMS) documento que traza una intención y un nuevo enfoque, el que conjuntamente con iniciativas y acciones, puede permitir instaurar una forma diferente de abordar y resolver los problemas de salud a nivel global. $^{2}$

El presente trabajo tiene como objetiv o realizar un acercamiento desde la perspectiva teórica al análisis del modelo de DSS de la OMS en el que se destacan los determinantes estructurales, y den tro de estos el papel de los procesos de exclusión social como aspectos a tener en cuenta por su repercusión en la salud de grupos vulnerables. Se pretende argument ar la compresi ón del prob lema a part ir de la presentación de conceptos y modelos de exclusión social y exclusión en salud, lo que contribuirá a la vez al in cremento del conocimiento del tema para aquellos interesados. 


\section{EL PROBLEMA}

\section{Construcción social de la salud}

El concept o de sal ud ha evol ucionado d esde I a defi nición d e "ausen cia de enfermedad" a un concepto un tanto utópico formulado por la OMS en 1978 con las siguientes palabras: "La salud es un bien estar físico, mental y social total y no simplemente la au sencia de do lencias o en fermedades". ${ }^{3}$ Reci entemente, se han formulado otras definiciones en las que se incluyen aspectos subjetivos y objetivos como el "s entirse bie n", la "habilidad para la adapta ción" o la "capacidad de funcionamiento autónomo." 4

Con indepe ndencia del modo en que se de fina, I a comprensi ón del concepto de salud se dirig e hacia el lado positivo del proces o de enfe rmar, hacia el mantenimiento de las c apacidades, habilidades y la integridad emocional que hace al individuo sentirse bien e incorp orado plenamente a todas las esfe ras de la vida social, laboral y familiar.

En la salu d se ex presa la t endencia a la salutogénesis, hacia la búsq ueda y provisión de recursos de diversa naturaleza que proteg en contra la posibilidad de enfermar o permiten recuperar el estado de bienestar una vez perdido. El análisis conduce a prestar atención al polo de la vulnerabilidad o propensión a enfermar, el que considera todos aquellos factores que au mentan la probabilidad de la aparición de la enfermedad, que ponen en peligro o amenazan la con dición de salu d de las personas. Estos actores rebasan el enfoque biologicista o medicalizado del proceso salud enfermedad e introducen el importante papel de lo social y lo psicológ ico en la compren sión de la producción y constr ucción de la salud. Este es entonces un análisis mucho más comple jo y multidimensional (Martín Alfonso L, 2009. Consideraciones conceptuales sobre los determinantes sociales de la salud para la acción intersectoria I. Ponencia presen tada en el Taller I nternacional de Intersectorialidad en la práctica social. La Habana).

De modo que: ¿en vi rtud de qué habrí a que construir la salud? Consideramos que sería necesario analizar instancias y niveles tales como:

- La estructura social, económica y política de la soc iedad. Según Benach y otros, los principales determinantes que condicionan la salu d y la en fermedad derivan de los efectos de la desigualdad en la distribución del poder económico y social y sus remedios deben ser $\mathrm{p}$ olíticos (Benach $\mathrm{J}$ y Mu stelier $\mathrm{C}$. La salu d in separable de la política. En: Aprender a mirar la salud. Editorial El viejo topo;1995).

- La desigualdad en la dist ribución de los recursos, la s condiciones de pobreza, alimentación, vi vienda, higiene, la falta de acceso a los servicios de salud por inexistencia o a Itos cost os, condicion an desde la pr opia est ructura social la $\mathrm{s}$ desigualdades en salud. Es de cir, la influencia de la posición social ocupada por los actores y los mecanismos de re distribución de los recursos, el acceso al trabajo y a los servicios. La salud debe ser parte esencial de la voluntad política del estado y la sociedad en general y reflejarse en acciones y estrategias que la materialicen.

- Condiciones sociodemográfi cas. Las variab les demográf icas se mezclan con el componente social. El sexo biológico de riva e $\mathrm{n}$ una co nstrucción de género que influye en la salud. La edad se traduce en desventajas para los ancianos y los niños que se convierten en poblaciones más vuln erables, as í ocurre con las d iferentes ocupaciones laborales. 
- Redes de interacción grupal social, familiar y cultural. Las redes de interacción a nivel de grupos sociales en los qu e participan las person as con stituyen referentes comportamentales y cu Iturales de gran $v$ alor para la comprensión de la sal ud. También las desigualdades sociales se expresan a nivel de grupos, los qu e requieren intervenciones dife rentes. Por ejemplo, el grupo familiar se convierte en una de las principales fuentes de apoyo para la conservación y rec uperación de la salud de $s$ us miembros y en esta se or ganizan di ferentes bi enes y servi cios consumidos por la mis ma que traducen condiciones y calidad de $v$ ida particulares de cada familia. ${ }^{5}$

- Salu d biológica. L a i nfluencia del con junto de f actores sociales y ambien tales depende de la resistencia biológica del organismo. ${ }^{6}$

- Comportamientos y recursos person ales. El ser huma no es una u nidad biopsicosocial en el que las influencias de todas las condiciones sociales de vida se hacen subj etivas a través de su conforma ción psicológ ica indiv idual que permite desplegar la capacidad de un funcionamiento comportamental responsable o no con la salud. El estilo de vida se construye de manera individual y expresa el modo y las condiciones de vida de las personas así co mo el papel de función reguladora de la personalidad como $v$ ariable qu e in terviene en e I a frontamiento a la $v$ ida, propiciando salud. Es decir, se trata de ver en la comprensión de la salud no solo la resistencia biológica sino también la res istencia personal. (Mar tín Alfonso L, 2009. Consideraciones conceptuales sobre los determinantes sociales de la salud para la acción intersectoria I. Ponencia presen tada en el Taller I nternacional de Intersectorialidad en la práctica social. La Habana).

El carácter complejo del concepto y su compren sión en t oda su in tegralidad compromete a las d isciplinas de las ciencias biológicas, las ciencias sociales y las ciencias humanas, ${ }^{6}$ es muy impor tante aproximarnos a una conceptualización que exprese teóricamente esta naturaleza compleja y permita su traducción en acciones prácticas encargadas de la prod ucción so cial de la sa lud con la participación y aporte del cuerpo de conoci mientos de todas las disciplinas científicas imp licadas, sin esquematismos, ni absolutismos.

\section{Determinantes Sociales de la Salud}

El enfoque de los DSS emerge co mo un modo de resalt ar las limit aciones de las conceptualizaciones y las intervenciones orientadas básicamente a los riesgos de la enfermedad en los individuos.

Como hemos apuntado antes en relación con el concepto de salud, en este enfoque también son muy importantes las formulaciones y sus tentaciones teóricas a $p$ artir del sistema de conocimientos de diversas d isciplinas científicas necesarias para el accionar en salud pública, lo cual implica tener en cuen ta diferentes perspectivas. Se ha señalado la perspect iva económica, la social-comunitaria y comportamental, la antropológica-cultural, la política y la ética. ${ }^{7}$

Desde la perspectiva económica, es necesario enfatizar en la correlación entre salud y clase soc ial y entre la posición en el tr abajo y el estado de salud . La pobreza, guerra, racismo, desigual dad de género, programas de ajuste estructural, desplazamientos y migraciones, son identi ficados como determinantes de riesgo para la $s$ alud de las poblaciones. La perspectiva social comunita ria y comportamental, inserta a las personas en familias, grupos sociales y modos de vida. La evidencia de dispar idad social entre grupos, y e $\mathrm{n}$ los grupos se relac iona con desigualdades en salud y bienestar. La desigualdad social y eco nómica ejerce 
una i nfluencia directa sobre el contenido de las relaciones so ciales y sobre la densidad d e la cohesión social. Tambié $n$ se fundamenta en el estudio de las relaciones entre los co mportamientos de las personas y la aparición de problemas de salud.

Desde la perspectiva cultural, se hace énfa sis en el papel de las representaciones, actitudes y sistemas de explicaciones de las personas, relativos a las enfermedades y las diferencias introducidas por la multiculturalidad en los problemas de salud. La perspectiva política abarca el pap el del con texto sociopolítico y la voluntad de lo s gobiernos y los sistemas de salud en la generación de políticas y programas enfocados o no a las desigualdades de salud y a con tribuir a man tenerlas o erradicarlas, mientras que la pers pectiva ética se centra en la con sideración de los valores personales y las experiencias subjetivas en la salud de las personas, lo que destaca la salud como un valor y un derecho inalienable para todos.

Los DSS se definen co mo las condiciones so ciales en la s que las personas viven y trabajan, las características sociales dentro de las cu ales la v ida tiene lu gar. Los DSS apuntan a rasgos específicos del cont exto social que afecta la sa lud y lo $\mathrm{s}$ mecanismos por los cuales las condiciones so ciales se traducen e $\mathrm{n}$ impactos en la salud. ${ }^{8}$ Los modelos clásicos de determinantes de la salud han considerado el papel de lo social en la producción de la salu d, eso es innegable, sin embargo el enfoque de los determinantes sociales centra el énfasis en las causas que a ctúan desde el contexto so cioeconómico y macroestructural más general en que se desenvuelven las poblaciones y los individuos las cuales son fuente de las inequidades en salud..$^{8,9}$

Se han planteado varios modelos de DSS. Ha sido muy citado el de Dahlgren y Whitehead denominado de influencias distribuidas en capas, el que trata de ilustrar las interacciones entre los diferentes niveles de condiciones causales, desde el nivel general hacia niveles particulares y un ni vel singular, que contempla lo personal y específico. ${ }^{8,9}$

A partir de los diferentes modelos de DSS, se propuso por el Equipo de Equidad de la OMS como marco conceptual para la Comisión sobre Determinantes Sociales de la Salu d, un modelo sin tético represen tado en la figura 1. En este se destaca el papel del contexto social y político incluidas las instituciones políticas y los procesos económicos que dan a I ugar a un conj unto de $\mathrm{p}$ osiciones s ocioeconómicas desiguales.

Los grupos se estrati fican según los ni veles de ingreso, la educación, el estado profesional, el género, el grupo étnico, la cohesión social, así como la in clusión y la exclusión social. Estos mecanismos de estratificac ión socioeconómica se describen como determinantes estructurales de la salud o como fa ctores sociales determinantes de las desigualdades en salud. ${ }^{8,9}$

Las posiciones socioeconómi cas se traducen en dete rminantes específicos del estado de salud que genera una exposici ón y vulnerabilidad dif erenciales en factores que influyen en la salud. Estos so $\mathrm{n}$ los determinantes intermediarios, entre los que se encuentra $n$ condiciones materi ales como la sit uación laboral, de vivienda, disponibilidad de alimentos, circunstancias psicosociales, facto res conductuales. El sistema sa nitario en sí mismo debe comprenderse como un determinante intermediario..$^{8,9}$ 


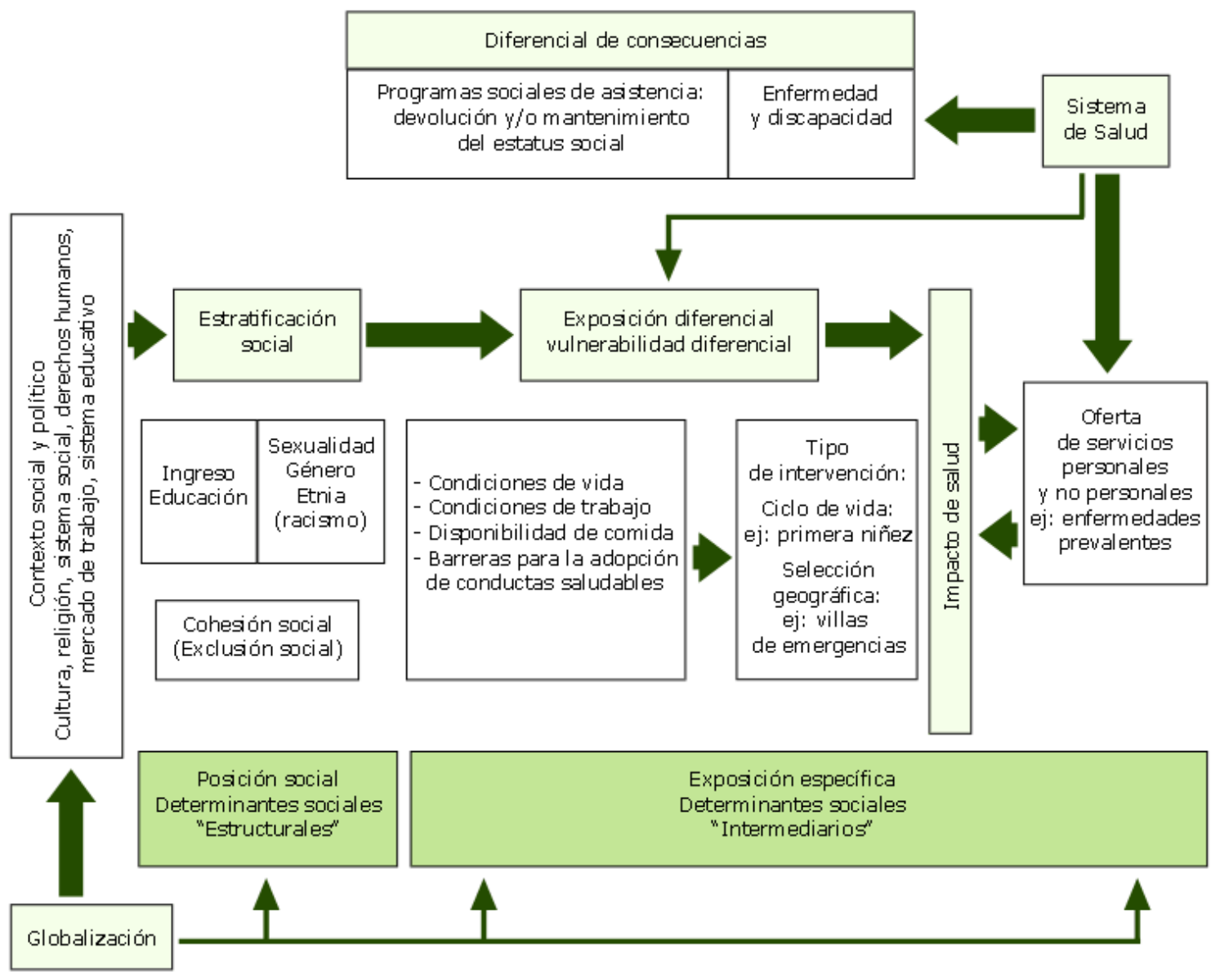

Fuente: ob.cit.8.

Fig. 1. Modelo de la Comisión de Determinantes Sociales de la Salud OMS.

\section{Exclusión social}

Los niños, adolescentes, mujeres, las pe rsonas con discapacidad, la población indígena, ru ral y aquella en situación de extrema pobreza, son rec onocidos como grupos tradicionalmente ex cluidos. ${ }^{10}$ Además de estos c olectivos, los inmigrantes han ocupado la atenció $n$ en programas de trabajo como personas en condición de exclusión social (Agost Feli p M. Excl usión social: una ex periencia de intervención sociolaboral. Proyecto Accord. Castellón de la Plana. 2004).

Diversas razones justifican la vulnerabilidad en los principales grupos de riesgo a la exclusión social. Las p ersonas con discapacidad física o psíquica pueden presentar dificultades de acceso a recursos psicos ociales, escas os recursos económicos o educativos, problemas sociales de incomunicación, soledad y aislamiento y si viven solos, pueden tener dificultades de seguir el tratamiento. Las personas sin techo, constituyen un grupo social sin recursos económicos, no tienen hogar, ni apo yos sociales, tienen problemas de alcoholismo u otras adiccion es, aislam iento, marginación e in visibilidad. Son u n colect ivo con grav es proble mas de ex clusión social al que, habitua Imente, las in stituciones socia les consideran con esca sas posibilidades de solución y se limitan a of recerles a lbergues o comedores sociales para cubrir sus necesidades básicas. ${ }^{10}$ 
Los inmigrantes son un grupo espe cialmente vulnerable. El proceso m igratorio está ligado, gen eralmente, a con diciones precaria s qu e af ectará el est ado de salu d influenciado por fac tores ambientales, ec onómicos, sanitarios y de género, e ntre otros.

Los ancianos son personas muy $v$ ulnerables por dist intas razones, entre estas, pueden car ecer de apoyo social por vivir solos o ais lados, presentar lim itaciones físicas (incapacidades, minusvalías ) o care ncias sociales y culturales. También en los desempleados de larga duración se generan problemas soci ales, económicos y personales, que los pone como grupos en riesgo de exclusión. Otros grupos sociales vulnerables en este sentido son los drogod ependientes, los enfermos de VIH/sida, los que ejercen la prostitución, los alcohólicos y los expresidiarios. ${ }^{10,11}$

La in fluencia de la Un ión Eu ropea en la gen eralización del con cepto de exclusión social ha sido remarc ada por numerosos in vestigadores sociales, así como la primera aparición del término en 1989 en el II Programa Europeo d e Lucha contra la Pobreza. Progresivamente, el término exclusión social se fue consolidando en documentos (Comisión Europea, 1993 y 19 94, respectivamente) en los que se enfatizó el carácter estructural de este proceso y se caracterizó por su dinamismo y multidimensionalidad, dada su conexión no solo con las situaciones de desempleo y rentas insuficientes sino con los pr oblemas de vivienda, niveles y oportunidades de educación, salud, y la integración social. Esta su stitución progresiva ha producido abundantes

aportaciones sobre la distinción y similitud de los conceptos pobreza y exclusión. ${ }^{12}$

Pobreza y exclusión social so n conceptos que se han empleado i ndistintamente, se han diferenciado, se han utilizado como complementarios sin que exista claramente un conse nso sobre su distinción o s imilitud. Se atrib uye el acu ñamiento del concepto exclusión social al francés René Lenoir en su obra "Les Exclus: un Français sur dix" (1975), donde estimaba que uno de cada diez franceses podía considerarse excluido e identifico incluso los grupos sociales en cuestión. ${ }^{12}$

La exclusión social se define como un proces o mediante el cual los individuos o los grupos son total o parcialmente excluidos de una participación plena en la sociedad en la que viven, como los obstáculos qu e encuentran determinadas personas para participar plenamente en la vida so cial, viéndose privadas de una o varias opc iones consideradas fundamentales para el desarrollo humano. ${ }^{12}$

El concepto de exclusión social es mu Itidimensional, y sus dimensiones pertenecen fundamentalmente a tres áreas: lo s recursos económicos, las re laciones sociales y los derechos legales. En cuanto a la d imensión económica, se señalan los ingresos insuficientes, la falta de acceso a los re cursos, la inseguridad en el empleo o el desempleo. La dimensión social s e refier e a la ruptura de los laz os sociales y familiares, fuente de capi tal social y de mecanismos de solidarid ad orgánica y comunitaria, la marginación social, la alteración de los comportamientos sociales, la falta de participación en las actividades sociales y políticas y el deterioro de salud. La dimensión política se expresa en la carencia de poder, la falta de participación en las decisiones que afectan la vida cotidiana, Ia au sencia de part icipación política y escasa representatividad. ${ }^{10}$

Los procesos de exclusión social presen tan dimension es e incluyen los factores personales, subjetivos y ps icológicos. El mo delo de García Roca, ${ }^{13}$ se caracte riza por presentar una dimensión estructural o económica identificada por la carencia de recursos $\mathrm{m}$ ateriales, derivada de la exclusión del mercado de trabajo, una dimensión context ual o soci al, especificada por la falta de in tegración en la $v$ ida familiar y en la comunidad de pertenen cia y una di mensión subjeti va o personal 
donde aparecen rupturas en la comunicación, debilitamiento de sentimientos vitales y síntomas de escasa satisfacción con la vida. ${ }^{13}$

Entre los mecanismos estructurales de prod ucción de la exclusión se encuentra la persistencia del dese mpleo de larga du ración, las con secuencias para el merca do laboral de los cambios en las sociedades in dustriales, el deterioro de las estructuras familiares, I a ev olución y las ru pturas del sist ema de valores, la t endencia a la fragmentación social y I a evolución de los fe nómenos migratorios (Agost Felip M. Exclusión s ocial: una experi encia de i ntervención sociolaboral. Proyecto Accord. Castellón de la Plana. 2004).

Una diferencia muy generalizada es reservar la palabra pobreza para las situaciones de carencia económica y material mi entras que el uso de la expres ión exclusión social designa un $\mathrm{pr}$ oceso de carácter es tructural qu e con siste en lim itar sensiblemente el acceso a un $\mathrm{n}$ úmero considerable de personas a un grupo de bienes y oportunidades vitales. Es decir, mientras la pobreza se restringe a una sola dimensión, I a económi ca; I a excl usión i ntegra una pluralidad de di mensiones (laboral, cultural, política y de salud). Se ha destacado que las ciencias sociales han ido adoptando progresivamente el concepto de exclusión social sin abandonar el de pobreza, marginación y privación. ${ }^{12}$

La exclusión social es un concep to y un fenómeno aú n en con strucción para las ciencias sociales contemporáneas, existe dispersión de acepciones que denotan diferentes comprensi ones para un mismo proceso. Se ha comparado el concepto con otros cercanos co mo marginación, segr egación, pobreza, y se con cluye que el de exclusión cumple una función integradora y de síntesis, dado que intenta unificar la dimensión econ ómica, la cu Itural, la social y la po lítica en u n solo $\mathrm{m}$ arco de análisis conceptual. ${ }^{12}$

En el campo de las ciencias sociales se manejan términos como la i nserción, la integración social, la promoción social y la inclusión social, como un modo de hacer frente a la pobrez a y la ex clusión. Cada uno de estos términos, tienen sus conceptualizaciones las que se tienden a manejar de manera predominante en dependencia del contexto.

Por otra parte, se de be señalar la te ndencia creciente a emple ar sistemas de indicadores sociales $\mathrm{p}$ ara la med ición de la exclusión social. Al r especto existen varias prop uestas ya sea en sentido amplio o para colectivos específicos como la infancia. ${ }^{12}$

A continuación centraremos el análisis del proceso de exclusión social en el modelo de García Roca, ${ }^{13,14}$ por ser un mo delo que destaca, además de los ejes económic 0 y social, un eje personal refe rido a las manifestaciones de orden psicológico, emocional, comport amental y de percep ción de as pectos personales vit ales implicados en I os procesos de excl usión soci al. Esta es una d imensión que consideramos importante reconocer y destacar en el análisis de este problema a fin de lograr una visión integral del ser humano y su grupo en situación de desventaja social.

Para an alizar el proce so de ex clusión socia I, comple jo y mu ltidimensional, García Roca, ${ }^{13,14}$ concretamente propone el análisis de tres dimensiones: la econ ómica, la social y la personal (Fig. 2), c uya ruptura da lugar a las situaciones de exclusión. Del mismo modo, considerando la exclusión como proceso, no podemos centrarnos solamente en aquellos que han llegado a las est aciones finales del itinerario, sino también en los que se encuentran recorriendo un camino u otro, configurándose así tres zonas en este recorrido y, por ex tensión en el espaci o social: zona de 
integración o coh esión, z ona de vu Inerabilidad y zona de margin ación o ex clusión (Agost Felip M. Exclusión so cial: una experiencia de intervención sociolaboral. Proyecto Accord. Castellón de la Plana. 2004).

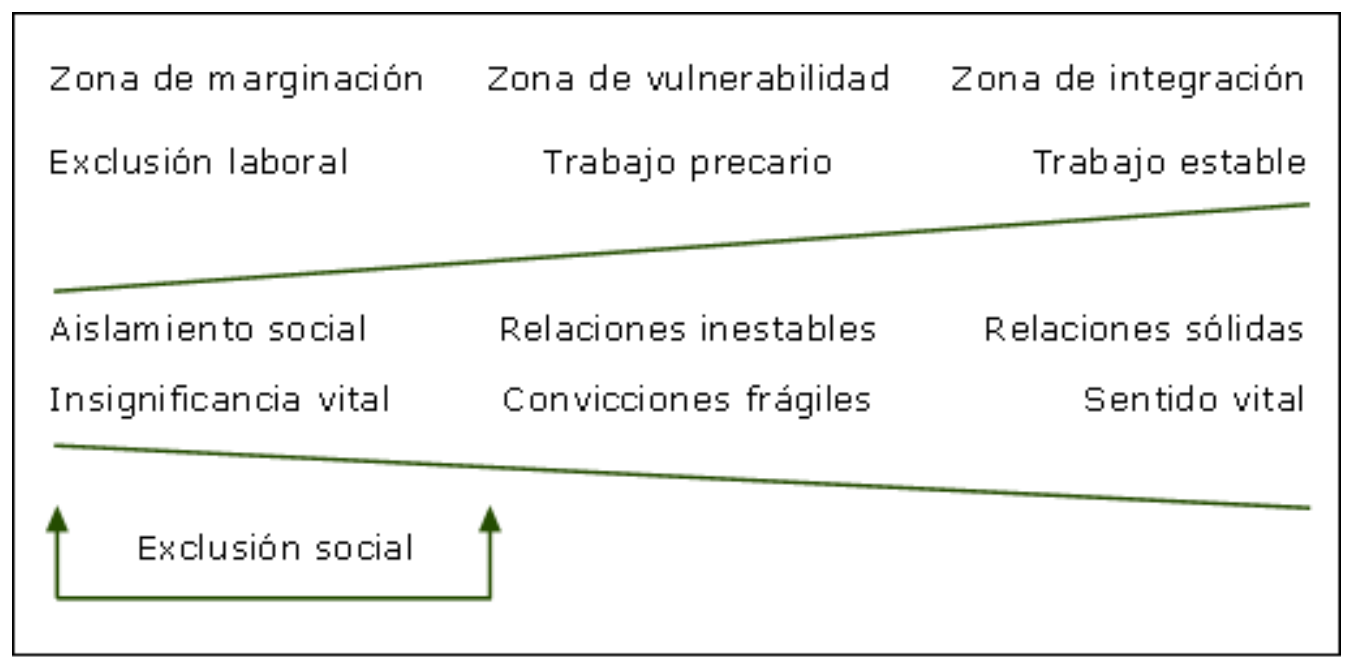

Fig. 2. Dimensiones y zonas del proceso de exclusión social.

El primer $v$ ector está compuesto por el ementos estructurales y se refie re a la dimensión económica de la ma rginación en un sistema de organizaci ón social que expulsa a grandes mayorías de beneficiar ios, el autor I a denomi na excl usión laboral. La zona de la ex clusión tiene h oy unas se ndas evidentes en el paro, la distribución de la renta y la desprotección so cial. Los indicadores por antonomasia son las conmociones acontecidas en el $\mathrm{m}$ undo del emp leo: escasez, precaried ad, final de l e mpleo y en la esfera de la distribución. Existen dife rentes factores que contribuyen a empeorar la s ituación que en relación con el empleo tienen muchas personas de los país es desarrollados, existen colectivos cuyos sectores se encuentran en reconversión, por lo que son susceptibles de entrar en un proceso de inestabilidad y vulnerabilidad; $t$ ambién existe una bolsa de pers onas que por diferentes motivos han quedado excluidas del sistema productivo, como los jóvenes que todavía no han accedido al $\mathrm{p}$ rimer emp leo, los parados de larga duración o personas con algún tipo de discapacidad.

El segundo vector está compuesto por elementos contextuales, se ref iere a la dimensión social de la marginación. Esta segunda dimensión está caracterizada por la diso lución de los $v$ ínculos social es, la $f$ alta de $f$ iliación y la $f$ ragilidad del entramado relacional. ${ }^{13} \mathrm{~A}$ esta dimensión responden las estrategias de accesibilidad por la vía social, como el incremento de la red de apoyo social, la creación de vínculos y el senti miento de $p$ ertenencia a una com unidad, en tre otras. $L$ os indicadores por excelencia son las conm ociones acontecidas en la estructura familiar, la movilidad social, las redes sociales.

El tercer vector está co mpuesto por elementos subjetivos. Comprende la dimensión personal de la marg inación, que para el autor, ${ }^{14}$ se traduce en la precar iedad cultural caracterizada por la rup tura de la comunicación, la debilidad de las expectativas y la er osión de I os din amismos $v$ itales ( confianza, iden tidad, reciprocidad), q ue co nllevan senti mientos de i ncompetencia y una menor participación e in tegración socia I. L os indicadores por antonom asia son las conmociones hoy acontecidas en la su bjetividad, la f ragilidad cultural para of recer sentido a la vi da, el desánimo y el debili tamiento de la con fianza, iden tidad y autoestima. ${ }^{13}$ 
Con respecto a las zonas del proceso de ex clusión, podemos decir que cada una de estas se caracteriza por el desplieg ue de tres referentes que afectan la relación con el $t$ rabajo, la re lación con las $v$ inculaciones $v$ itales $y$ la re lación con las significaciones que otorgan sentidos y motivaciones para vivir. Cada uno de los tres referentes se confi gura como un continuum que va desde el trabajo fijo, las vinculaciones est ables y los sign ificados plenos (zo na de la cohesión) hasta el desempleo, la ruptura de las vinculaciones y el sinsentido de las motivaciones (zona de ex clusión), pasando por la $z$ ona in termedia de la $v$ ulnerabilidad, en la que el trabajo, las relaciones sociales y las significaciones $v$ itales se $r$ ealizan de man era precaria, inestable y frágil. ${ }^{13}$

A continuación, un análisis más detallado de cada una de las tres zonas del proceso de exclusión:

\section{- Zona de cohesión o integración social}

La llamada sociedad industrial se basa en cuatro principios de inclusión, gracias a los cuales se ha mante nido cohesionada du rante los dos últimos siglos: el trab ajo productivo como principio de inclu sión económica; el co nsumo como principio de inclusión s ocial; la pr otección pú blica como prin cipio de in clusión polít ica y la competitividad como prin cipio de in clusión cu Itural. ${ }^{14}$ Así pu es, la z ona qu e podemos considerar cohesionada de la soc iedad ha sido aquella form ada por todas aquellas personas con empleo estable, con capacidad de consumir bienes sociales, protegidas ante los diferentes riesgos y que pueden competir en la vida.

\section{- Zona de vulnerabilidad}

Desde mediados de la década de los ochenta, se han tambaleado todos y cada u no de los principios de integración establ ecidos por la sociedad industrial, su convulsión ha sido de tal grado que nada ni nadie se ha podido librar d e la turbulencia. $Y$ de este modo, ha aparecido una nueva vulnerabilidad de masas. ${ }^{14}$

- Las convulsiones ac ontecidas en el mundo laboral (pre carización del empleo y el paro), la convulsión del desarrollo co nsumista (demostraci ón de progreso económico y limitación de los recu rsos del plan eta) y las con vulsiones del E stado del Bienestar, son los tres pilares so bre los que se sustenta la zona de vulnerabilidad den tro de los procesos de ex clusión social. Junto a e stas convulsiones laborales, del consumo y del Estado, el individualismo se suma co mo un importante factor de vulnerabilidad. Por una parte, la emergencia del sujeto y su autonomía pero, por o tra parte, significa también la presencia de una cul tura que persigue el propio interés y desconfía de todas las formas colectivas de pertenencia.

\section{- Zona de exclusión}

Tres circuitos resultan decisivos para entender la forma actual en la que se produce la exclusión, cada uno de los cuales es como una onda expansiva sobre el resto de la sociedad: los factores estructurales, que conforman una organización excluyente, los factores contextuales, que cristalizan en contextos deshabilitantes y los factores motivacionales, que hacen frágil los dinamismos vitales. ${ }^{13}$

Las zonas del itinerario de exclusión han sido recreadas en un continuo que va de la integración a la ex clusión. L a primera es la $z$ ona de in tegración en la que se situarían aquel los individuos que cuentan c on un trab ajo estable y un si stema relacional sóli do tanto a ni vel familiar como vecinal. La segunda es una zona de vulnerabilidad $y$, por lo $\mathrm{t}$ anto, in estable, caract erizada por la precariedad, los 
trabajos temporales, el desemp leo y en el plano relacional, por la fragilidad de los soportes familiares y sociales. Finalmente, la zona de marginalidad o exclusión que se caracteriza por la a usencia de trabaj o y aislamiento social (Agost Fel ip MR. Economía social y desarrollo. Material docente. Máster Universitario de Cooperación al Desarrollo. Universidad Jaume I. 2010.).

Algunos au tores consideran cru ciales las ev entuales in tervenciones pú blicas (asistenciales o de inserción ) así como la u bicación de las person as en redes sociales que proporcio nen ayudas o estímulo $\mathrm{s}$ a la inserción a la $\mathrm{h}$ ora de transitar desde una zona de $v$ ulnerabilidad hacia la integración o hacia la exclusión (Agost Felip MR. Economí a soci al y desarroll o. Ma terial docente. Mást er Universitario de Cooperación al Desarrollo. Universidad Jaume I. 2010).

\section{Exclusión social y salud}

Como ya se ha comentado, I a exclusión social se considera un determinante social estructural que determina desigualdades en salud. Esta se ha i ntentado vi ncular con problemas de salud específicos, por ejemplo con las enfermedades cardiovasculares, sobre las que se plantea la interrogante de si la exclusión social juega un papel determinante en la elevada incidencia de muerte súbi ta cardiaca en la era actual y s i factores de índole políticos son de abor daje impostergable en virtud de lograr resu Itados $\mathrm{f}$ avorables en I a dismin ución de la morbi lidad y mortalidad por estas e nfermedades; se ap unta que los costos por concepto de atención médica de urgencia ante una dolencia cardiaca, y la toma de decisiones en relación con cond uctas de intervención, II evan aparejado la condición social de quien la sufre. ${ }^{15}$

También se señ ala la exclusión social como un co ncepto útil para comprender I as experiencias sociales de aquellos con problemas de salud mental y la necesidad urgente de un trabajo más conceptual y metodológico en este terreno. ${ }^{16}$

Desde hace al gunas décadas, Ia OMS y Ia OPS han trabajado la exclusión en salud en el terreno teóri co, metodol ógico y es tratégico. A los efectos se definió la exclusión en salud como "la falta de acceso de ciertos grupos o personas a diversos bienes, servicios y oportunidades que mejoran o preservan el estado de salud y que otros i ndividuos y gr upos de I a soci edad di sfrutan". De este enunci ado se desprende que se trat a de un fenómeno qu e involucra al sector de la salud y trasciende en él. ${ }^{17}$

A partir de la definición, se señ alaron las sigu ientes proposiciones: la exclusión en salud es una entidad distinguible y susceptible de caracterizar, es posible identificar indicadores para medirla, puede a su vez ser utilizada como una medida del éxito o fracaso de las políticas destinadas a mejorar la situación de salud, y los sistemas de protección de salud no son neutrales respecto de I a excl usión si no que, por el contrario, pueden dete rminar diversos grados de esta al interior de un sistema de salud. ${ }^{17}$

La exclusión en salud puede aparecer liga discriminación racial y a otras formas de da a la pobrez a, la margin alidad, la culturales que incluyen el idioma, la informalidad en el e mpleo, el subempleo y e desempleo, el ais lamiento geográfico, en es pecial ligado a la a la vid a del campo y a su s labores; la f alta de serv icios bá sicos como elec tricidad, agua potable y saneamiento básico y un bajo nivel de educación o información de los us uarios de los servicios. 
Una de las manifestaciones de la exclusión social, definida como la falta de acceso a los bienes, las oportunidades y las relaciones sociales disfrutadas por otros, es la falta de acc eso a los servicios de salud, de este modo s e podría considerar que la exclusión en salud es una expresión de la ex clusión social, lo cual se refleja en la literatura en el tema que presenta casos de exclusión en salud como un ejemplo de exclusión social. Algunos incorporan el componente de salud dentro de su definición de exclusió n social. Por ejemp lo, para el Gobierno Inglés, ${ }^{17}$ exclusión social es " lo que puede ocurrir cuando las personas o las áreas s ufren de una combinación de problemas ligados entre sí como desemple o, falta de habilidades, bajo ingreso, viviendas pobres, ambientes de alt a crim inalidad, mal a salu d y qu iebra en la estructura familiar."

Sin embargo, existen aspectos de la ex clusión en sal ud que no conl levan el elemento de pérdida de la integración social que es central en la idea de exclusión social y que parece depender de va riables más propias del sector, co mo el modelo de provi sión de servici os, I a asignaci ón de recursos humanos, tecnológicos y financieros al in terior del sector y el grado de información de los usuarios de los servicios. De este modo se pudiera concebir la exclusión en salud como una entidad distinguible, que no es posible explicar solo a través de la pobreza, la inequidad o la exclusión social (Fig. 3).

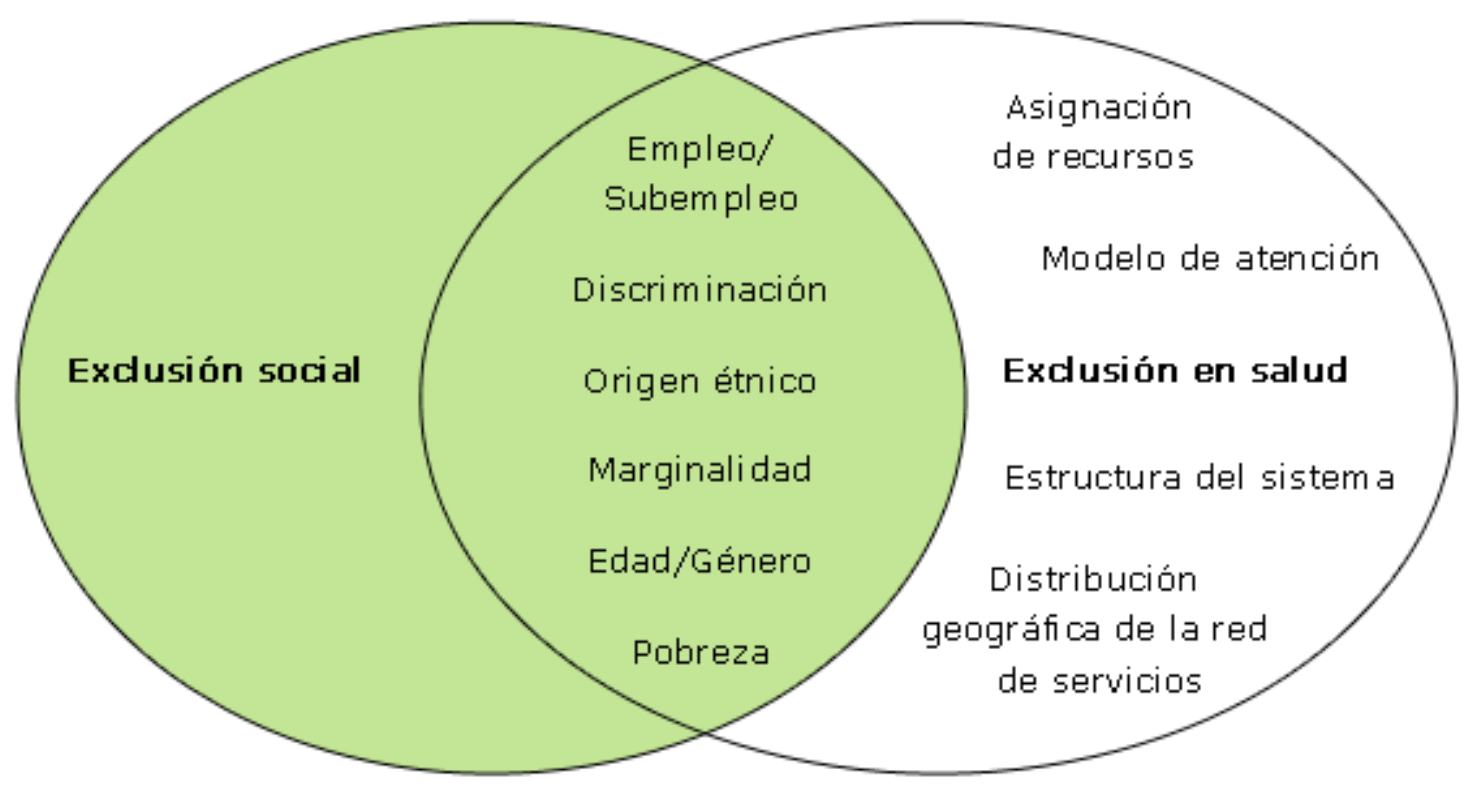

Fuente: Ob.cit. 17.

Fig. 3. Relación exclusión social y exclusión en salud.

Los estudios de caracterización de la exclusión en sal ud reali zados en di ferentes países de América Latina y el Caribe (República Dominicana, Guatemala, Honduras, Ecuador, Perú y Para guay) o frecen result ados que ilustran el panorama de este problema en sus respe ctivos contextos sociales, políticos y económicos. ${ }^{17}$ En estos se utilizó una guía metodológica para estudiar la ex clusión social en salud, la cu al constituyó una val iosa alternativa para el acercami ento a I a constatación y sistematización de información sobre el tema. ${ }^{18}$ 
Es de destacar, a los efectos de esta public ación, que en dichos estudios se manejó el concepto "exclusión soci al en salud", (integrando lo s términos social y salud), para referirse a "las fallas que se producen en los sistemas de pr otección social en salud" y así destacar el papel de las intervenciones públicas o de otros actores de la sociedad or ientadas a expandir la prote cción social que pued en con tribuir a la reducción de las exclusiones en salud. ${ }^{19}$

La protección social en salud, la otra cara de la moneda de la exclusión social en salud, se d efine como la garantía que los po deres públicos otorgan para que un individuo o grupo de individuos pueda sati sfacer sus demandas de salud y obtener acceso a los servicios en forma op ortuna y de manera adecuada a través de alguno de los subs istemas de salud exis tentes. Es ta defi nición consi dera el conjunto de mecanismos disponibles para que la pobl ación tenga acceso a la protección y cuidado de su salu d median te la asign ación de recursos de divers o origen y no solamente como las medidas que toma el Estado para asegurar la atención de salud a través de la provisión pública de servicios. Es importante anotar que la definición no solo se refiere a g arantizar acceso, sino tambi én calidad y oportunidad de la atención. ${ }^{20}$ La protección social en salud pued e ser definida como la garantía que los poderes públicos otorgan para que un individuo o grupo de ind ividuos pueda satisfacer sus demand as de salud, obteniendo acceso a servicios de buena calidad en forma oportuna. ${ }^{19}$

Se ha realizado un lla mado a examinar los sistemas de protección social en salud en los pa íses de Amér ica Latina d e manera que se tracen polít icas que realmente cubran las necesidades de salud de todos los grupos sociales. ${ }^{21}$ En este sentido es importante apuntar que el sistem a de prot ección social en salud, particularmente en Cuba, requiere un análisis especial, pues sus políticas y acciones han contribuido a preservar la salud d e la poblac ión a pesa $r$ de la crisi $s$ econ ómica vivida en la década de los noventa. El modo de enfrentamiento a la sit uación de cris is y su potencial $r$ epercusión en la salu d permit en con ocer las mejo res pol íticas y estrategias que se pueden utilizar para minimizar estos efectos. ${ }^{22}$

El mundo contemporáneo vi ve una al armante sit uación de cri sis econ ómica expresada de diferentes maneras, ámbitos y niveles de profundidad, no se avizoran con certeza mecanismos polít icos y soci ales a nivel global que contribuyan a resolver una situación que afecta cada vez m ás a todos. Por esto, los temas de protección, inclusión y su contrap arte, la exclusión social, co bran cada vez más relevancia y atención para quienes se ocupan de trabajar en función del bienestar y la salud de los seres humanos que habitan un planeta puesto en riesgo de magnitud creciente.

No es casual que el $t$ ema de los DSS se haya retomado con tan ta fuerza en los últimos años. Es importante y necesario investigar, proponer y ejecutar acciones, proyectos y estrategias que permitan reducir su impacto en la salud y la vida de las personas y los grupos. ${ }^{23}$

Por último, nos preguntamos ¿p or cuáles ví as la exclusión social influye en la salud?, ¿en cuáles pro blemas de salud ti ene mayor relevancia?, ¿en qué contextos se expresa de una forma y en cuáles de otra? Esto consti tuye un campo de indagación empírica susceptible de ser ex plorado y nutri rse con Ia constatación de datos a partir de la investigación. 


\section{REFERENCIAS BIBLIOGRÁFICAS}

1. Al meida Filho N, Silva Pai $n$ J. La crisis de I a salud pública y el movimiento de salud colectiva en Latinoamérica. Cuadernos Médico Sociales [Internet]. [citado 15 Sept 2011]. Disponible en: http://www.ilazarte.com.ar/cuadernos/pdf/n75a345.pdf

2. Comisión de Determinantes Sociales de la Salud. Declaración provisional. Lograr la equ idad en salu d: desde las ca usas in iciales a los re sultados ju stos. Gin ebra: OMS; 2005a.

3. Conferencia Mundial de Salud en Alma-Atá. Ginebra: OMS; 1978.

4. González Menéndez R. La psicología en el campo de la salud y la enfermedad. La Habana: Editorial Científico-Técnica; 2004.

5. Louro I. La familia en la det erminación de la salu d. Rev Cuba na Salud Pública [Internet]. 2003 [citado 20 Sept 2009 ];29(1): 48-51. Disponible en: http://scielo.sld.cu/scielo.php?script=sci arttext\&pid=S0864$\underline{34662003000100007 \& \operatorname{lng}=e s \& n r m=i s o \& t \operatorname{lng}=e s}$

6. C onstandriopoulus A. E lementos para una topografía del concepto de salud. Universidad de Montreal. Revista Interdisciplinaria Salud. 2006;11(1).

7. OPS- REDET. Cinco caras de I a nueva salud pública. Universidad de Mo ntreal. (PPT). Fundamentos de los D eterminantes Sociales de la Salud. Edición abril-julio. Washington, D.C.: OPS; 2009.

8. Comisión de Determinantes Sociales de la Salud. Hacia un marco conceptual que permita analizar y actuar sobre los determ inantes sociales de la salud. Documento de trabajo. Ginebra: OMS; 2005.

9. Moiso A. Determinantes de la salud. En: Barragán $\mathrm{HL}$, editor. Fundamentos de la Salud $\mathrm{P}$ ública. Argen tina, $\mathrm{L}$ a $\mathrm{P}$ lata: Un iversidad Nacional de La $\mathrm{P}$ lata, Editorial EDULP; 2007.

10. Del Campo G. Apuntes: So ciología d e la salud. Exclusión social y salud [Internet]. Universidad de Alicante; 2011 [citado 15 May 2011]. Disponible en: http://www.aniorte-nic.net/progr asignat sociolog salud.htm

11. Es tébanez $E$ stébanez $P$. E xclusión so cial y salud. Balance y perspectivas [Internet]. Barcelona: Icaria E ditorial, SA; 2002 [citado 20 Sept 20 11]. Disponible en: http://books.google.es/books

12. Hernández Pedreño M. Exclusión social y de sigualdad. Murcia: Universidad de Murcia, Servicio de publicaciones; 2008.

13. García Roca J . Lo público y lo privado frente a la ex clusión social: prácticas, discursos y narraciones. Madrid: HOAC; 1993.

14. . Contr acultura d e la solida ridad y Exclusión so discursos y narraciones. Madrid: HOAC; 1998.

cial: prácticas , 
15. Ochoa Montes LA . Exclusión social y muerte súbi ta. Rev Cuba na Salud Pública [Internet]. 2010 citado $20 \mathrm{~S}$ ept 200 9];36(3). Disponible en: http://scielo.sld.cu/scielo.php?script=sci arttext\&pid=S0864$\underline{34662010000300012 \& \operatorname{lng}=e s \& n r m=i s o \& \operatorname{lng}=e s}$

16. Morgan C, Burns T, Fitzpatrick R, Pinfold V, Priebe $S$. Excl usión social y salud mental: Revisión conceptual y metodológica. Br J Psychiat. 2007;191(6).

17. Exclusión en salud en países de Améric a Latina y el Caribe. Serie Extensión de la protección social en salud. Washington, D.C.: OPS; 2003.

18. Gu ía met odológica para la caracterización de la exclusión social en salud . Unidad de Desarrollo de P olíticas y Sist emas de Salu d. Wash ington: OPS/ OMS; 2006.

19. M inisterio de Sa lud Pública y Asistencia Social/ Dirección Gen eral de I Sist ema Integral de Atención en Salud. Protección social en salud. Representación OPS/OMS en Guatemala: OPS/OMS; 2002.

20. E studio Naci onal de Excl usión en Sal ud en Hondura s. Protec ción y excl usión social en salud Internet]. Washington: OPS; 2003[citado 20 Sept 2009]. Disponible en:

http://new.paho.org/hq/dmdocuments/2010/Exclusion Salud Honduras 2003.pdf

21. Rosenberg $H$, And ersson B. Repensar I a protección social en salud en Améric a Latina y el Caribe. Rev Panam Salud Pública. 2000;8(1/2).

22. Hadad Hadad J L, Valdés Llanes E. Protección social en salud c omo enfrentamiento a una crisis económica. Rev Cuba na Salud Pública [Internet]. 2010 [citado 20 Sept 2009];36( 3). Disponible en:

http://scielo.sld.cu/scielo.php?script=sci arttext\&pid=S0864-

34662010000300008\&lng=es\&nrm=iso\&tlng=es

23. Santos Padrón $\mathrm{H}$. Los determinantes so ciales, las desigualdades en salud y las políticas como temas de investigación. Rev Cubana Salud Pública [Internet]. 2011 [citado 20 Sept 2009];37( 2). Disponible en:

http://scielo.sld.cu/scielo.php?script=sci arttext\&pid=S0864-

34662011000200007\&lng=es\&nrm=iso\&tlng=es

Recibido: 10 de octubre de 2011.

Aprobado: 21 de octubre de 2011.

María Raquel Agost Felip. U niversidad Jaume I. Castellón de La Plana, España. Correo electrónico: ragost@psi.uji.es

* Este artículo es fruto de la estancia de Investigación en la convocatoria 2011 del Plan de P romoción de la In vestigación de la Un iversidad J aume I. E spaña. Se enmarca en el proyecto de investigación "Análisis del apoyo social y del capital social" código 10I395.05 (Plan de Promoción de la investigación UJI-Bancaja). 